\title{
MORTALIDAD EN ALPACAS EN ÉPOCA DE ESTIAJE Y SU EFECTO \\ ECONÓMICO EN LOS PRODUCTORES EN LA COMUNIDAD DE HUAYTIRE PROVINCIA DE CANDARAVE - TACNA.
}

Responsable: MVZ. Cecilio Hurtado Quispe Miembro: MVZ. Cesario Cruz Anchapuri

\section{RESUMEN}

El estudio realizado comprende la mortalidad en alpacas de la raza huacaya, en sus diferentes clases, en la época de estiaje, Se seleccionaron 10 productores de la zona y se realizaron visitas mensuales a los productores para registrar los datos de mortalidad por clase de animal. Los resultados obtenidos para crias $16,6 \%$, tuis de un año $6,21 \%$, machos $4,76 \%$, hembras $5,52 \%$, con un promedio general de $9,14 \%$ de mortalidad por rebaño familiar. Una descapitalización del rebaño familiar del 9,7\%. con una reducción del ingreso económico familiar del $16,20 \%$. Se observó que la mayor pérdida económica corresponde por mortalidad de hembras con $48 \%$, seguido por tuis $26 \%$, crias $18 \%$ y machos $8 \%$.

\section{ABSTRACT}

The study includes mortality in the race huacaya alpacas, in their different classes, at the time of drought, 10 were selected producers in the region and reralizaron monthly visits to producers to record mortality data from animal class, the results for offspring $16.6 \%$, tuis one year $6.21 \%$ males $4,76 \%$, females $5.52 \%$, with an overall average of $9.14 \%$ mortality family herd, a herd of decapitalization family of $9.7 \%$. with a reduction in family income of $16.20 \%$, it was noted that the biggest economic loss corresponds with female mortality by $48 \%$, followed by tuis $26 \%$ offspring $18 \%$ and males $8 \%$.

\section{INTRODUCCIÓN}

El estudio se realizó en el Centro Poblado Menor de Huaytire - provincia de Candarave, región de Tacna, teniendo como objetivo determinar la mortalidad de alpacas en la época de estiaje y el efecto económico en los productores de Huaytire.

La problemática en la crianza de alpacas, dentro de su proceso de crianza, está relacionada directamente con los factores de producción y medioambiente. El desequilibrio de esta interrelación tiene como efecto la baja productividad de fibra, carne y pieles.

En la crianza alpaquera las épocas de mayor preocupación son los meses denominados épocas de estiaje, siendo críticos los meses de mayo y septiembre, presentándose condiciones medioambientales severas, bajas temperaturas $\left(-28{ }^{\circ} \mathrm{C}\right)$, reducción de biomasa vegetal $(60 \%)$, insolaciones fuertes, vientos y heladas.

Según reportes en otras zonas en épocas de estiaje, las mortalidades alcanzan hasta un $30 \%$ del total de la población, de los cuales $60 \%$ son crias. Estas cifras tienen consecuencias en la reducción del capital pecuario, haciéndo crítico el ingreso económico familiar y siendo uno de los factores que induce a los productores al abandono de esta crianza, incrementando el nivel de migración y la oferta de mano de obra barata en las grandes ciudades.

\section{MATERIALYMÉTODOS}

Ubicación geográfica:El estudio se realizó en época de estiaje del 2007, en el Centro Poblado Menor de Huaytire, ubicado entre las coordenadas de Longitud Oeste $70^{\circ} 21^{\prime \prime}$ y Latitud Sur de $16^{\circ} 56^{\prime \prime}$, a una altitud de 4450 msnm perteneciente a la cuenca del río Locumba de la provincia de Candarave, región de Tacna,

El presente estudio tiene como objetivo determinar la mortalidad de alpacas en la época de estiaje y el efecto económico en los productores de Huaytire.

\section{Método:}

Selección de familias alpaqueras: Se ha seleccionado a 10 familias alpaqueras, el método de selección ha sido con el uso de padrones comunales, teniendo en consideración el número de alpacas por unidad familiar que son mayores de 80 animales, unidades familiares menores a esta cifra no se consideraron en la selección, por razones que tienen actividades complementarias y sus ingresos económicos son limitados con respecto a la producción alpaquera. También se tuvo en consideración la infraestructura 
productiva. Sólo se tomaron familias sin ninguna construcción de infraestructura moderna (cobertizos), con la finalidad de tener una muestra con iguales condiciones.

Trabajo de campo: Consistió en realizar visitas a cada uno de las unidades familiares en sus cabañas con el objeto de capacitar en el llenado de fichas de seguimiento de la mortalidad de su rebaño, estos datos fueron contrastados en las visitas mensuales de la época de estiaje.

Registro de mortalidad de alpacas: Para este efecto se ha elaborado una ficha de registro de datos, donde se registran clase animal, mes de mortalidad, capital total pecuario, en cada una de las familias seleccionadas.

Determinación de pérdidas económicas por mortalidad: A fin de conocer los costos de la fibra, carcasa y pieles se han realizado diferentes visitas a las ferias locales de la zona, especialmente las ferias semanales del distrito de Mazo Cruz, donde se efectúa el acopio constante de los recursos alpaqueros y los precios de estos insumos están actualizados, los mismos que sirvieron para la determinación de la pérdida económica de las familias alpaqueras de Huaytire.

\section{RESULTADOSY DISCUSIONN}

\section{Composición y reducción del rebaño familiar:}

\begin{tabular}{|c|c|c|c|c|c|c|c|}
\hline \multirow[b]{2}{*}{$\mathbf{N}^{0}$ familia } & \multicolumn{2}{|c|}{ Adultos } & \multirow{2}{*}{ Tuis } & \multirow{2}{*}{ Crias } & \multirow{2}{*}{ Total } & \multirow{2}{*}{$\frac{\mathrm{N}^{\circ}}{\text { Mort }}$} & \multirow{2}{*}{$\frac{\%}{\text { Reduc }}$} \\
\hline & Hembras & Machos & & & & & \\
\hline 1 & 68 & 19 & 35 & 31 & 153 & 20 & 13.1 \\
\hline 2 & 40 & 6 & 22 & 19 & 87 & 8 & 9,2 \\
\hline 3 & 55 & 15 & 30 & 33 & 133 & 15 & 11,3 \\
\hline 4 & 35 & 6 & 22 & 19 & 82 & 8 & 9,8 \\
\hline 5 & 43 & 4 & 27 & 24 & 98 & 10 & 10,2 \\
\hline 6 & 52 & 9 & 26 & 22 & 109 & 9 & 8.3 \\
\hline 7 & 51 & 6 & 32 & 35 & 124 & 8 & 6,5 \\
\hline 8 & 48 & 2 & 26 & 23 & 99 & 8 & 8,1 \\
\hline 9 & 54 & 5 & 32 & 35 & 126 & 10 & 7,9 \\
\hline 10 & 70 & 8 & 35 & 41 & 154 & 17 & 11,0 \\
\hline Total & 516 & 80 & 287 & 282 & 1165 & 113 & 9,7 \\
\hline
\end{tabular}

En la Tabla 1. se observa el total del capital pecuario de cada rebaño familiar, distribuido por clase animal en un rango de 82 animales hasta 154 animales por rebaño; estas variaciones son debido al recurso disponible con que cuenta cada productor y la reducción del rebaño por efectos de mortalidad de los animales, lo cual se observó desde $7,9 \%$ a $13,1 \%$, con un promedio general de $9,7 \%$, por efectos medioambientales en la época de estiaje.

Estudios realizados en diferentes comunidades campesinas de la zona sur de Puno, provincia de EI Collao, por parte de PAL/PRATEC (1989), reportan que las inclemencias medioambientales afectan significativamente a los componentes hombre-animal, el productor alpaquero es afectado por la reducción de sus ingresos económicos, mientras que el componente animal es afectado en la descapitalización pecuaria, siendo mayor en agosto por alta presentación de friaje, con mortalidades hasta en un $40 \%$ y reducción de ingreso económicos del 30 al $50 \%$. Mientras que para nuestro estudio alcanzó un promedio general del $9,14 \%$ de mortalidad por rebaño familiar; esta diferencia se debería a la época estacional de muestreo en que ha sido realizada, considerando que cuando se efectuó nuestro trabajo no se presentó friaje de consideración, lo que ocasionó un menor porcentaje $(9,14 \%)$ de mortalidad.

Hallazgos cualitativos obtenidos para la región de Puno, por Fernández, S. (1970), reportaron que la investigación en países en vias en desarrollo, por la poca disponibilidad de recursos de los productores, a menudo origina una serie de problemas de tipo social y económico, entre los que cuentan la mala nutrición, migración urbana, problemas que se asocian a la falta de incentivos a la producción agropecuaria. Estos hallazgos cuanlitativos tienen mucha similitud con los reportados en nuestro estudio debido a que se ubica en similar altitud y en las mismas condiciones ecológicas y medioambientales.

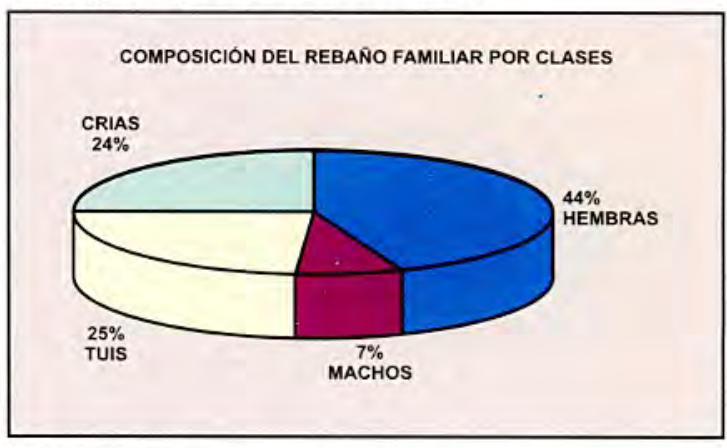

Figura 1. Distribución de alpacas por clase. Los datos corresponden al promedio de las 10 familias seleccionadas.

En la figura 1, se muestran los resultados de la composición del rebaño familiar. Para animales hembras se tiene el $44 \%$, animales machos $7 \%$, tuis entre machos y hembras $25 \%$ y para crías machos y hembras el $24 \%$.

\section{Mortalidad de alpacas:}

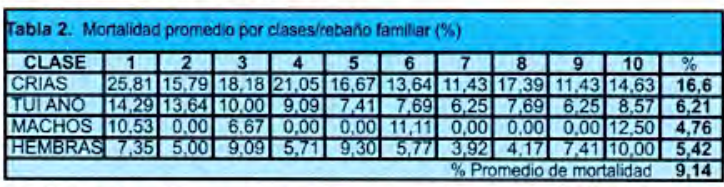

Tabla 2. La presentación de mortalidad por clase animal. Se observó que en crías las mortalidades por rebaño familiar son muy variadas, de: $11,43 \%$ a $25,81 \%$, con un 
promedio para esta clase de $16,6 \%$. Para animales de la clase tuis un año se observa de: $6,25 \%$ a $14,29 \%$, con un promedio general para esta clase de $6,21 \%$. Para el caso de animales machos existe una variación desde $0,00 \%$ a $12,50 \%$, con promedio general para esta clase de $4,76 \%$, indicando que el número de animales machos es muy mínimo dentro del rebaño familiar puesto que son los de esta clase que son destinados para la saca. En animales hembras existe una mortalidad de $5,71 \%$ a $10 \%$, con el promedio general para esta clase de $5,42 \%$, con un promedio general del $9.14 \%$ de mortalidad por rebaño familiar.

Estudios realizados sobre la producción y productividad en alpacas en la región de Arequipa, por Álvarez, J. (1982), reportarón una mortalidad en crías del $17 \%$ y una mortalidad total de $11,68 \%$, natalidad de $60-71 \%$, vientres: $49,11 \%$, saca: $21,87 \%$. Estos resultados encontrados son similares a nuestro estudio de $16,6 \%$ de mortalidad para crias de alpaca, mientras que la mortalidad general para alpaca es de $9,14 \%$; esta similitud tendría relación directa con la similar altitud entre ambas zonas de estudio y la coincidencia que ambos trabajos se realizaron en época de estiaje.

Similares trabajos efectuados en Puno, por ECHO TPS. (2002), reportaron que en épocas de friaje se presento una pérdida de $10,4 \%$ de mortalidad en hatos familiares con un $19 \%$ de abortos en animales preñadas; alcanzando una reducción del ingreso per cápita de 33,05\% por efectos de bajas temperaturas. Estos resultados también corroboran nuestros hallazgos encontrados $(9,14 \%)$, debido a que también se realizaron en época de estiaje.

Estudios reportados para la región de Arequipa, por CISA. (1990) hacen referencia a los hallazgo encontrados en diferente épocas del año, indican que el periodo más crítico en la explotación de alpacas está en los periodos secos después de las lluvias, afectando a los animales crías y a las hembras en gestación y esta última con abortos que en muchos caso llega hasta el $60 \%$.

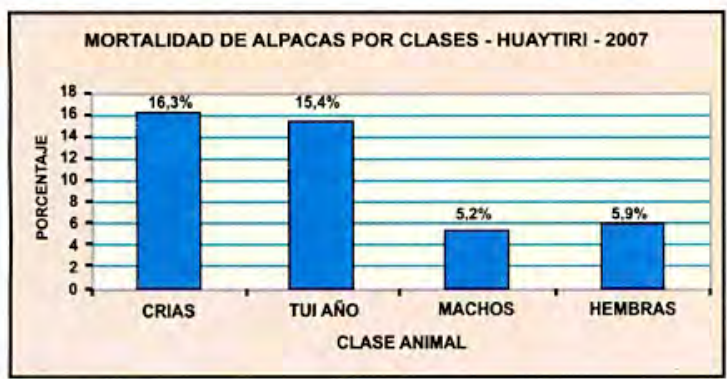

Figura 2. Mortalidad de alpacas por clases, la clase animal más afectado son crias y tuis, los machos y hembras alcanzan una similitud.
En la figura 2. Los animales más afectados fueron de la clase crias con $16,3 \%$. tuis $15,4 \%$ seguido por la clase hembras de $5,9 \%$ y los animales machos alcanzaron el 5,2\%

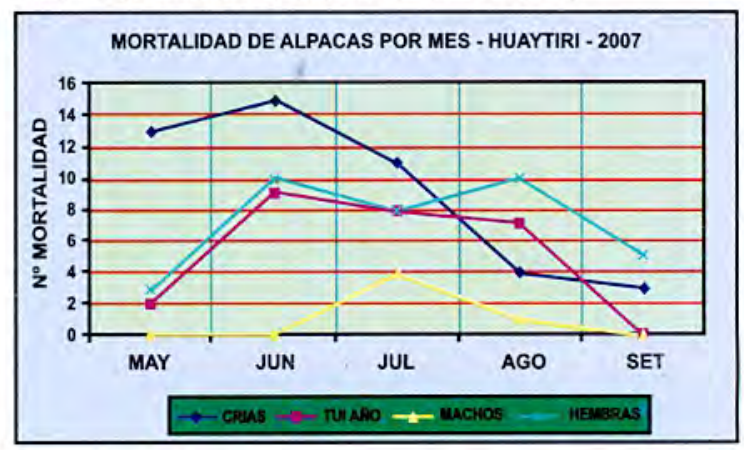

Figura 3. Mortalidad mensual, una alta tasa de mortalidad se observó en crias en el mes de junio, con comportamiento similar en tuis $y$ hembras, en machos en el mes de julio.

En la figura 3. El comportamiento de la tasa de mortalidad en diferentes clases en la época de estiaje se observó para la clase animal crías en el mes de junio, con similar comportamiento para las clases tuis y hembras, en lo que respecta para animales machos se observa en el mes de julio, y a partir de agosto las tasas de mortalidades decrecen gradualmente concordando con los cambios medio ambientales que existen, un incremento de temperatura, humedad iniciando las primeras lluvias.

\section{Pérdidas económicas por mortalidad de alpacas en épocas de estiaje:}

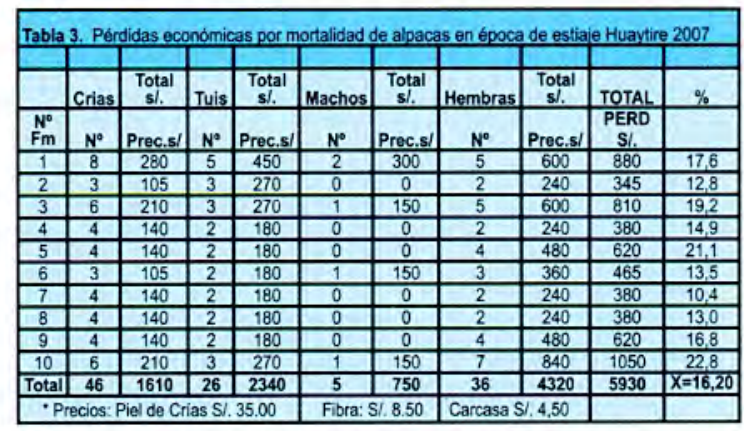

Tabla 3. Durante la época de estiaje se observaron pérdidas económicas por causa de mortalidad de alpacas en un rango de 345,00 a 1050,00 nuevos soles en las familias alpaqueras, produciendo la reducción de sus ingresos económicos desde 10,4 a $17,6 \%$ con un promedio general de $16,20 \%$, indicador que solo corresponde a la época de estiaje, no se consideran otras épocas del año.

Estudios realizados en comunidades de Puno por ECHO TPS (2002), reportaron una pérdida de ingreso per cápita de $33,05 \%$ en los criadores alpaqueros por efectos de friaje en épocas de estiaje. Este resultado difiere del 
hallazgo encontrado en nuestro estudio de $16,20 \%$ de ingreso per cápita en el productor alpaquero de Huaytire, debido a que la zona de estudio en Puno sufrió con mayor intensidad el friaje y por más tiempo, afectando mayormente al productor alpaquero de Puno.

Otro estudio realizado en Puno, en las comunidades campesinas de la zona sur de la provincia de El Collao, por parte de PAL/PRATEC, (1989), reportó una reducción de ingreso per cápita del 30 al $50 \%$ en estas comunidades campesinas. Estos resultados varian grandemente con los hallazgos encontrados en nuestro estudio de $16,20 \%$, debido a que los reportados para Puno se efectuaron en varias épocas del año, y nuestro resultado solamente para una época del año.

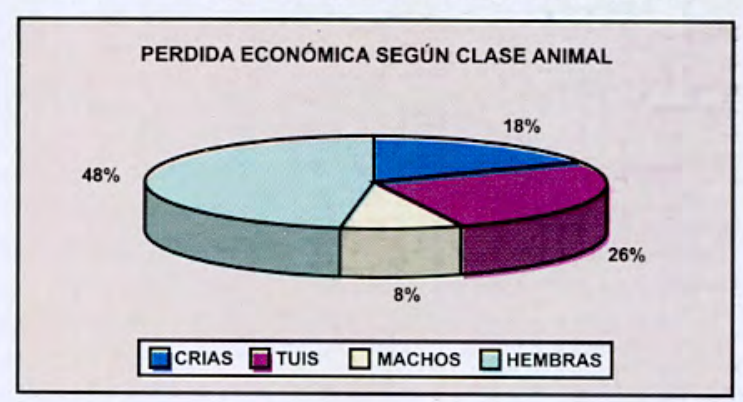

Figura 4. Pérdida económica según clase animal, las pérdidas económicas dentro del rebaño familiar provienen por mortalidad de animales hembra.

En la figura 4 se observa que la mayor pérdida económica corresponde a la mortalidad de animales hembras con $48 \%$, seguido por tuis $26 \%$, crías $18 \%$ y machos $8 \%$. Estos indicadores están relacionados con la edad, peso, precio y número de animales, en caso de las hembras se muestra mayor pérdida porque esta clase animal; corresponde por ser la población significativa dentro de la población del rebaño, en caso de los machos se reporta una pérdida poco significativa, porque la mayor población son destinados a la saca.

PAL/COTESU (1984) La crianza de alpacas es una actividad de suma importancia económica para el poblador altoandino, debido a la capacidad de la alpaca para adaptarse a grandes altitudes, pero todavia no existe un procedimiento que se este aplicando como un sistema concreto. El tipo de crianza no es definido, no siendo empírico, ni técnico, lo que existe es una combinación no definida de ambos puntos de vista, siendo los sistemas aún inadecuados a la especie, resultando así una productividad muy baja.

\section{CONCLUSIONES}

1. La mortalidad en alpacas en épocas de estiaje en Huaytire alcanza el $9,14 \%$, del total del rebaño familiar y por clase de animal son las crías más vulnerables con $16,6 \%$.

2. Durante la época de estiaje del año 2007 , se observó una reducción del capital pecuario del $9,7 \%$, con una pérdida económica de $16,20 \%$ menos en el ingreso per capita, familiar. Los animales que reportaron mayor perdida económica son los animales hembras, con $48 \%$ en relación a otras clases.

\section{RECOMENDACIONES}

Realizar investigaciones sobre sistemas de comercialización y transformación de insumos primarios (Fibra, Carne, Pieles).

Realizar evaluaciones de los humedales altoandinos para conocer la soportabilidad y capacidad de carga.

Identificar niveles tecnológicos de los productores en diferentes lugares de producción alpaquera de Tacna.

Evaluar la cobertura vegetal y composición vegetal de los humedales altoandinos.

\section{VI.BIBLIOGRAFÍA}

PAL/PRATEC. 1989. Crianza de llamas y alpacas, Proyecto Alpacas. Puno.

Coordinadota Interinstitucional del Sector Alpaquero Cusco 1990. Tecnología y cultura en la producción alpaquera.

ECHO TPS - 2002. Emergencia en altura, Proyecto de apoyo a las poblaciones afectadas por las tormentas de nieve y bajas temperaturas.

Fernandez, S. 1970. Crianza de camélidos domésticos IVITA. Cuzco.

Calle, R. 1980. Explotación de alpacas en centro del Perú-Junín.

Álvarez, J. 1982. Producción y productividad en alpacas. Arequipa 